\title{
Disruption of p16 and Activation of Kras in Pancreas Increase Ductal Adenocarcinoma Formation and Metastasis in vivo
}

\author{
Wanglong Qiu', Fikret Sahin ${ }^{3,4}$, Christine A. Iacobuzio-Donahue ${ }^{3}$, Dario Garcia- \\ Carracedo ${ }^{1}$, Wendy M. Wang ${ }^{1}$, Chia-Yu Kuo ${ }^{1}$, Doris Chen ${ }^{1}$, Dan E. Arking ${ }^{5}$, Andrew \\ M. Lowy ${ }^{6}$, Ralph H. Hruban ${ }^{3}$, Helen E. Remotti ${ }^{2}$, and Gloria H. Su, ${ }^{1,2 *}$ \\ ${ }^{1}$ The Departments of Otolaryngology and Head and Neck Surgery, Columbia University College of Physicians and Surgeons, \\ New York, NY 10032 \\ 2 Department of Pathology, Columbia University College of Physicians and Surgeons, New York, NY 10032 \\ 3 The Sol Goldman Pancreatic Cancer research Center, Department of Pathology, The Johns Hopkins University Medical \\ Institutions, Baltimore, MD 21205 \\ ${ }^{4}$ Microbiology Department, School of Medicine, Ankara University, Turkey \\ ${ }^{5}$ McKusick-Nathans Institute of Genetic Medicine, Johns Hopkins University School of Medicine, Baltimore, MD 21205 \\ ${ }^{6}$ Department of Surgery, Division of Surgical Oncology, Moores Cancer Center, University of California, San Diego, La Jolla, \\ CA 92093-0987 \\ Correspondence to: Gloria H. Su, email: gs2157@columbia.edu
}

Keywords: p16, LOH at Kras, pancreatic cancer, metastasis, mouse model, conditional knock-out, target therapy

Received: November 16,2011, Accepted: November 22, 2011, Published: November 23, 2011

Copyright: ( $)$ Qiu et al. This is an open-access article distributed under the terms of the Creative Commons Attribution License, which permits unrestricted use, distribution, and reproduction in any medium, provided the original author and source are credited.

\section{ABSTRACT:}

Inactivation of tumor suppressor gene p16/INK4A and oncogenic activation of KRAS occur in almost all pancreatic cancers. To better understand the roles of $p 16$ in pancreatic tumorigenesis, we created a conditional p16 knockout mouse line $\left(p 16^{f l o x} / f l o x\right)$, in which $p 16$ is specifically disrupted in a tissue-specific manner without affecting p19/ARF expression. p16 flox/flox; LSL-Kras ${ }^{G 12 D}$; Pdx1-Cre mice developed the full spectrum of pancreatic intraepithelial neoplasia (mPanIN) lesions, pancreatic ductal adenocarcinoma (PDA), and metastases were observed in all the mice. Here we report a mouse model that simulates human pancreatic tumorigenesis at both genetic and histologic levels and is ideal for studies of metastasis. During the progression from primary tumors to metastases, the wildtype allele of Kras was progressively lost (loss of heterozygosity at Kras or LOH at Kras) in p16fox/flox; LSL- Kras ${ }^{G 12 D}$; Pdx1-Cre mice. These observations suggest a role for Kras beyond tumor initiation. In vitro assays performed with cancer cell lines derived from primary pancreatic tumors of these mice showed that cancer cells with LOH at Kras exhibited more aggressive phenotypes than those retained the wild-type Kras allele, indicating that LOH at Kras can provide cancer cells functional growth advantages and promote metastasis. Increased LOH at KRAS was also observed in progression of human pancreatic primary tumors to metastases, again supporting a role for the KRAS gene in cancer metastasis. This finding has potential translational implications- future KRAS target therapies may need to consider targeting oncogenic KRAS specifically without inhibiting wild-type KRAS function.

\section{INTRODUCTION}

Pancreatic cancer is the $5^{\text {th }}$ leading cause of cancer deaths in the United States. The 5-year survival rate is only $5 \%$, despite significant increases in our understanding of the molecular events that mediate its onset and progression [1]. More reliable early detection methods are needed, as well as increased understanding of the mechanisms of metastasis and therapeutic resistance [2].

$K R A S$ is one of the most frequently activated oncogenes in human tumors, with $95 \%$ mutation 
frequency in pancreatic carcinomas [3, 4]. Point mutations in codon 12 of KRAS (KRAS G12D or KRAS $S^{G 12 V}$ ) are the most commonly identified in human pancreatic adenocarcinoma. This and other mutations that activate $K R A S$ have been found in the earliest stages of pancreatic intraepithelial neoplasia (PanIN)-a precursor to infiltrating pancreatic ductal adenocarcinoma (PDA). PanIN can be divided into different grades (1A, 1B, 2 and 3) according to the degree of architectural and cytological atypia [5]. This association between KRAS mutations and PanIN, along with data from in vitro transformation assays, support the concept that pancreatic tumorigenesis can be initiated by activated KRAS.

Cellular responses to Ras activation vary and depend on the cell type, Ras isoform, expression level, and wild-type allele status $[6,7]$. For example, targeted overexpression of activated Kras in the pancreas often leads to the development of pancreatic acinar hyperplasia or dysplasia, but not invasive cancer [8, 9]. Conversely, expression of activated $\operatorname{Kras}^{G 12 D}$ at a physiological level (in LSL-Kras ${ }^{G 12 D}$ mice), induced by Pdx1-Cre or p48-Cre, is sufficient to initiate the development of murine PanIN, some of which progress to PDA after a period of latency $[10,11]$. The oncogenic function of mutant Kras protein is therefore significantly influenced by the expression level of the mutant Kras allele or the ratio of the wild-type to mutant Kras protein. Increasing evidence supports the latter hypothesis that the wild-type Kras allele can also impact cellular responses [7, 12, 13].

A human PDA sample contains an average of 63 genetic alterations [14]. Among them, p16 (INK4A) is inactivation in virtually all human PDA [15]. The
A

$$
\text { C }
$$

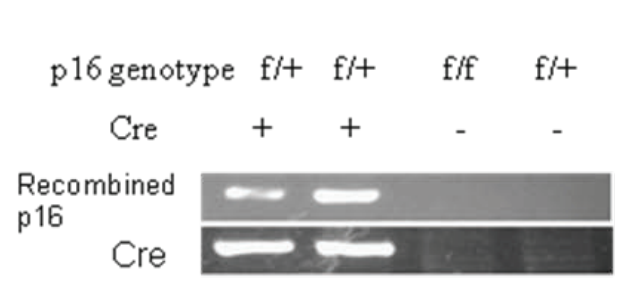

B



D

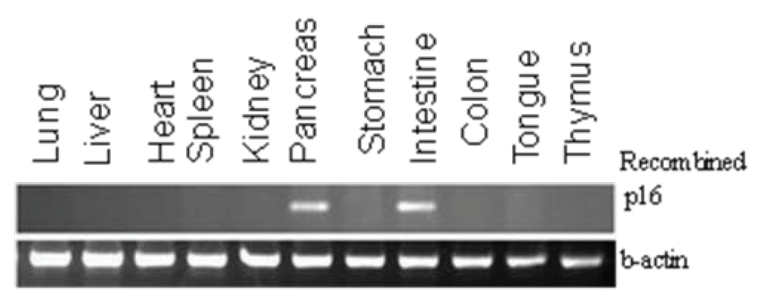

\section{$E$}

$H \& E$
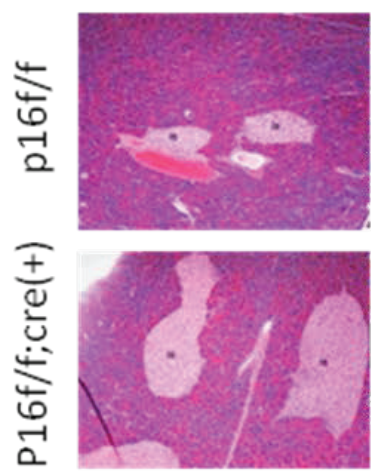

Glucagon


Insulin
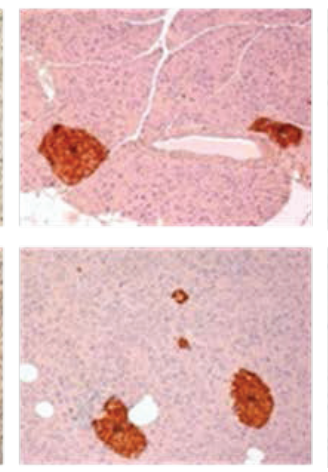

p16

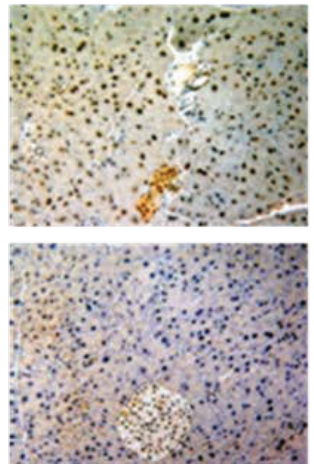

Figure 1: Tissue-specific inactivation of p16 in mice. (A) Schematic of the PCR strategies for genotyping and detecting Cre-

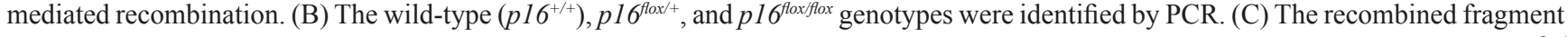
of $p 16$ was detected only in mice with Cre transgene expression, and in the pancreas and intestine, but not in the other tissues of adult $p 16^{f t o x}$

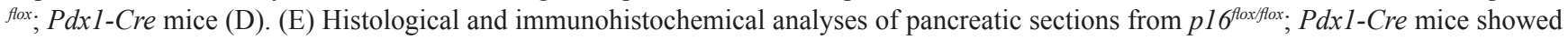
normal development of pancreatic cell lineages with significant reduction of p16 protein expression. 
molecular mechanisms of p16 inactivation include promoter methylation, missense mutation, and small deletion accompanied by loss of heterozygosity (LOH) $[15,16]$. Other than small deletions, methylation and missense mutations specifically targeted $p 16$ and did not involve $p 14(A R F)$, another tumor-suppressor gene with distinctive protein structure and function but shares coding exons with p16 [15-20]. In mice, activation of Kras alone is sufficient to induce the development of preinvasive lesions [11], and the deletion of the $p 16 / 19$ (INK4A/ARF) locus rapidly advances progression to invasive cancer as observed in LSL- Kras ${ }^{G 12 D}$; p16/19-/; Pdx1-Cre mice [18, $21]$. However, the role of $p 16$ in pancreatic tumorigenesis cannot be separated from that of $p 19$ in this model [18, 21]. $p 16^{--}$mice (which retain $p 19 / A R F$ ) spontaneously develop and die from a variety of malignant tumors, including soft-tissue sarcoma, lymphoma, and melanoma [22], so the potential tumorigenic effects of p16 loss in the pancreas are not known. To investigate the role of $p 16$ in pancreatic tumorigenesis, the most frequently mutated gene in human PDA, we generated conditional p16 knockout mice. In these mice only exon $1 \alpha$ of the $p 16 / 19$ locus is flanked by loxP sites, to inactivate only the $p 16$ allele in a tissue-specific manner without affecting the expression of $\mathrm{p} 19$.

\section{RESULTS}

\section{Targeted deletion of p16 in the pancreas does not affect development of pancreatic cell lineages}

In $p 16^{\text {floxfflox }}$ mice, exon $1 \alpha$ of both alleles of $p 16$ alleles are flanked by loxP sites (Fig. 1). Mutant $p 16^{\text {flox } f \text { flox }}$; $P d x$ 1-Cre mice were born at normal frequency and had no evidence of gross anatomic or physiological abnormalities. In adult $p 16^{\text {floxfflox}} ; \mathrm{Pdx} 1$-Cre mice, tissue-specific genomic recombination of $p 16$ was restricted to the pancreas and intestine (Fig. 1D). The expression levels of amylase, insulin, and glucagon in the mutant mice appeared normal (Fig. 1E and data not shown). Immunohistochemical analysis demonstrated the loss of the p16 nuclear labeling in all three pancreatic cell lineages in the $p 16^{\text {flox } f f o x} ; P d x 1$ -

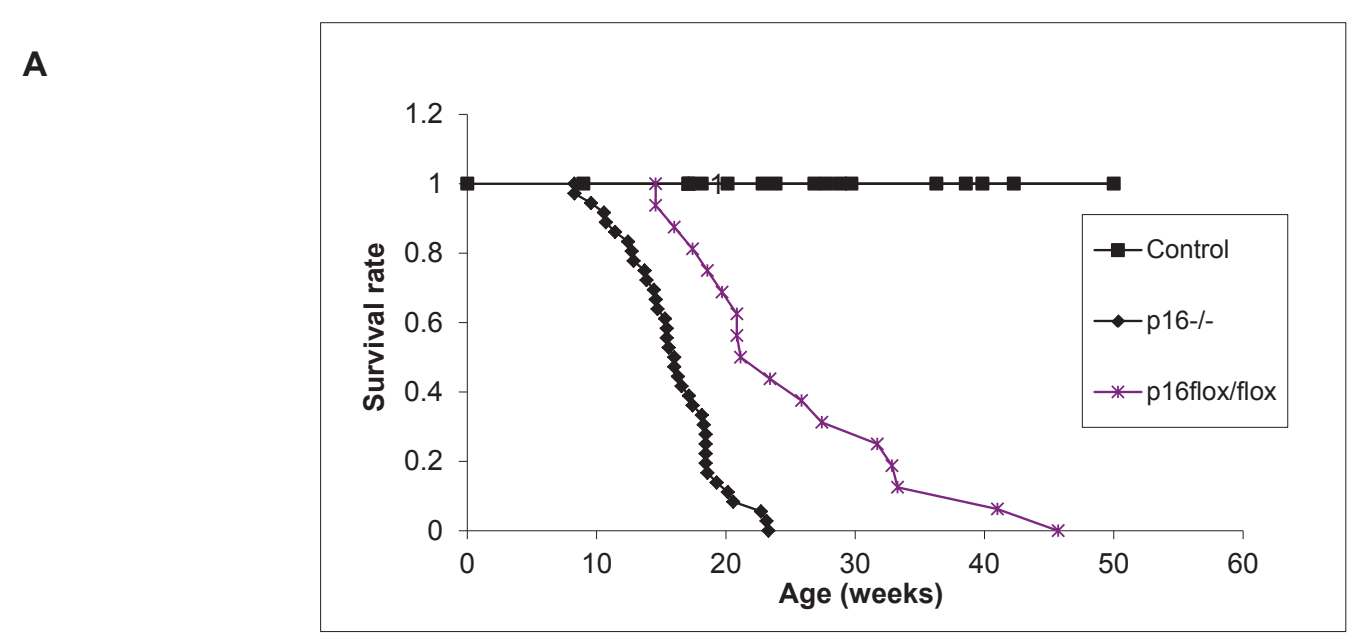

B
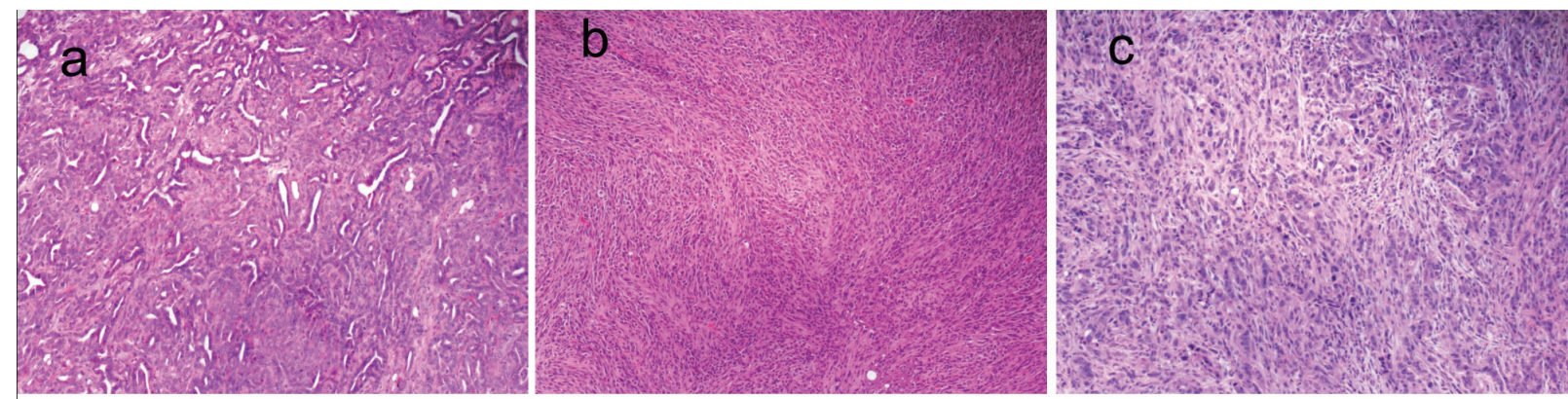

Figure 2: Effects of $p 16$ inactivation and Kras activation on survival and pancreatic tumor development. (A) The median survival time was $15.5 \pm 3.8$ weeks $(\mathrm{n}=36)$ for $p 16^{-}$; LSL-Kras ${ }^{G 12 D}$; Pdxl-Cre mice and $25.5 \pm 8.9$ weeks (n=16) for $p 16^{\text {fox } f f o x}$; LSL-Kras ${ }^{G 12 D}$; Pdxl-Cre mice. The control mice were mixed genotypes without the LSL-Kras ${ }^{G 12 D}$ allele; $\mathrm{n}=115$. (B) The most common histologic variant in the $p 16^{--} ; L S L-K r a s^{G I 2 D} ; P d x 1-C r e$ and $p 16^{\text {floxffox }} ;$ LSL-Kras ${ }^{G I 2 D} ;$ Pdx 1-Cre mouse models was comprised predominantly of malignant glands characterizing as well to moderately differentiated adenocarcinoma (a) with a minor component composed of more poorly differentiated components of sarcomatoid (b) and undifferentiated anaplastic (c) carcinoma. Shown here are representative histology from $p 16^{--} ;$LSL-Kras ${ }^{G 12 D} ;$ Pdxl-Cre mice. 
Cre mice, while diffuse nuclear labeling for p16 was observed in the pancreas of the $p 16^{\text {flox } f f o x}$ control mice (Fig. $1 \mathrm{E})$. The mutant and control mice had similar responses to the glucose tolerance test (data not shown). Finally, none of the $p 16^{\text {flox/flox}} ; \mathrm{Pdx} 1$-Cre mice under 18 months of age $(\mathrm{n}=16)$ developed pancreatic neoplasms, therefore $p 16$ inactivation alone is not sufficient to initiate pancreatic tumorigenesis.

\section{Combined p16 inactivation and Kras activation promote pancreatic tumor progression and metastasis}

To explore the dynamics of combined p16 biallelic inactivation and Kras activation in pancreatic tumor development and progression in the setting of intact p19, p16- ; LSL- Kras ${ }^{G 12 D}$; Pdx1-Cre mice and $p 16^{f 10 x}$ fox; LSL- Kras ${ }^{G 12 D} ;$ Pdxl-Cre mice were generated and characterized. $p 16^{-} ; L S L-\mathrm{Kras}^{G 12 D} ; \mathrm{Pdx1}$-Cre mice and p16 $6^{\text {Atx ffox }}$; LSL-Kras ${ }^{G 12 D} ;$ Pdxl-Cre mice had dramatically reduced median survival time ( $15.5 \pm 3.8$ weeks, $\mathrm{n}=36$, and $25.5 \pm 8.9$ weeks, $\mathrm{n}=16$, respectively) compared with pl6 ${ }^{++}$; LSL- Kras ${ }^{G 12 D}$; Pdxl-Cre mice ( 15 months) [11] (Fig. 2A).

Other than the differences in median survival, the $p 16^{-} ;$LSL-Kras ${ }^{G 12 D} ;$ Pdxl-Cre and $p 16^{\text {foxffox}}$; LSL$\mathrm{Kras}^{G 12 D}$; Pdx1-Cre mice were very similar in their tumor development and metastasis patterns. Mice of the $p 16^{-/}$; $L S L-K r a s^{G 12 D}$; Pdxl-Cre genotype were normal until 6 weeks of age. Between 6 and 24 weeks of age, they started to exhibit weight loss, jaundice, ascites, and increased abdominal girth (Fig. S2). A series of autopsies revealed the presence of solid pancreatic tumors ranging from 3 to $30 \mathrm{~mm}$ in diameter. The tumors were grossly firm with irregular and ill-defined margins, frequently adherent to
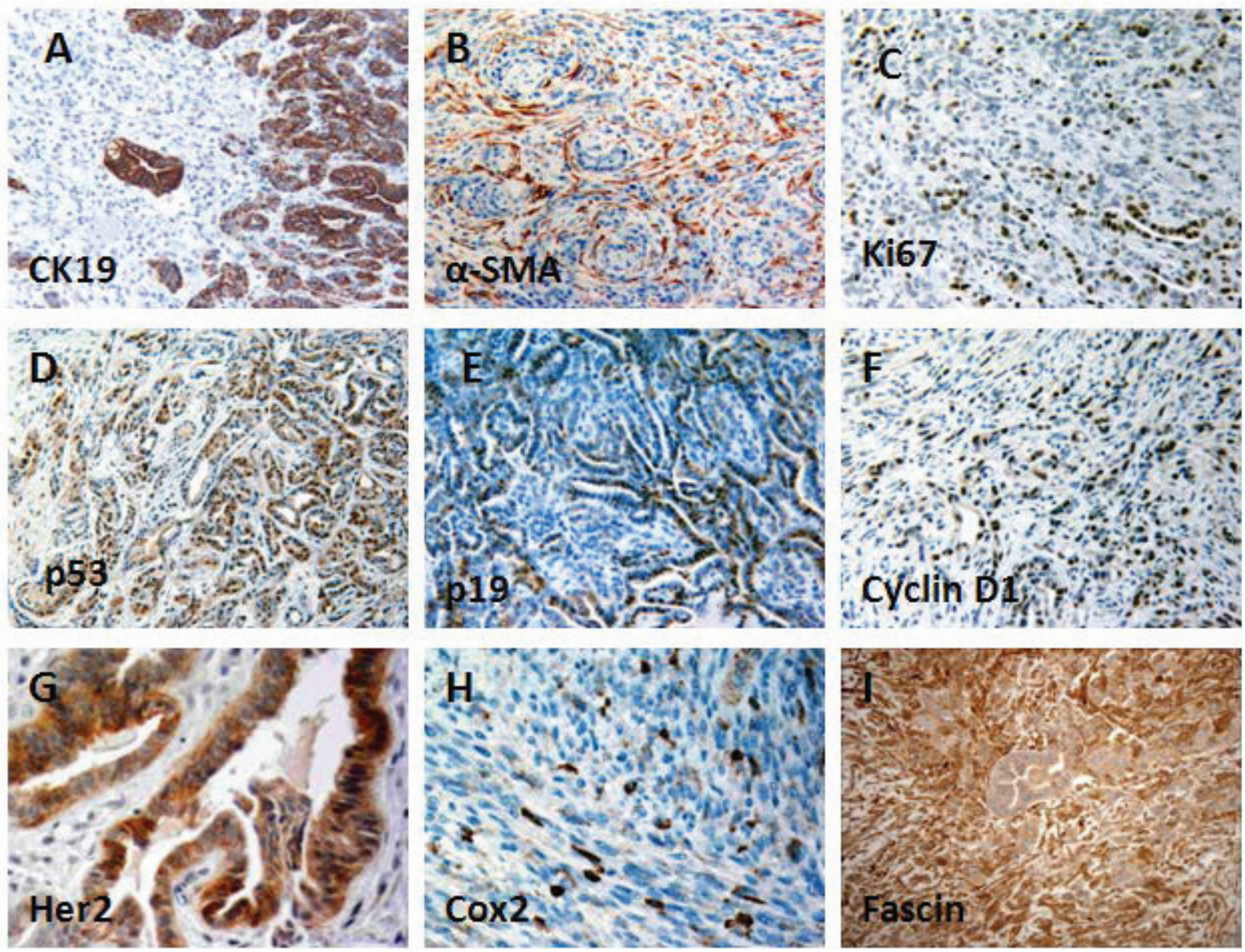

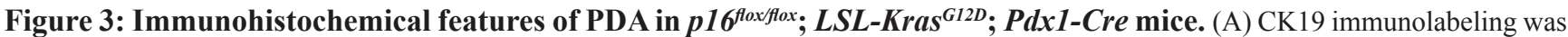
strong and diffuse in well-differentiated tumor, but negligible in the poorly differentiated or undifferentiated components; (B) Myofibroblasts, identified by $\alpha$-SMA immunostaining, were associated with a desmoplastic stromal response and were commonly associated with invasive pancreatic cancer; (C) PDA cells showed increased proliferation, compared with normal tissue, based on increased nuclear staining of Ki67; Nuclear expressions of p53 (D) and p19 (E) were detected in PDA. Cyclin D1 (F), Her-2 (G), and Cox-2 (H), and Fascin (I) were also overexpressed in PDA. 
adjacent organs (Fig. S2). Multiple tumor nodules were often visible, indicating that neoplasms of this genotype were multifocal. Histological analysis revealed a glandular pattern of well-differentiated to moderately differentiated pancreatic adenocarcinomas resembling human PDA was the predominant histologic component observed in both mouse models. Adenocarcinomas were identified in 21 of 22 invasive carcinomas in $16^{-/} ; L S L-\operatorname{Kras}^{G 12 D}$; $P d x 1-C r e$ mice and comprised $\geq 50 \%$ in 13 of the 21 invasive carcinomas (Fig. 2B-a, Table S1) and in 9 of 9 in $p 16^{\text {floxfflox}} ; \mathrm{LSL}-\mathrm{Kras}^{G 12 D} ; \mathrm{Pdx} 1-\mathrm{Cre}$ mice and comprised

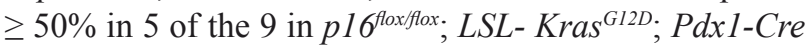
mice (Fig. S3f, Table S2). Undifferentiated spindle cell and undifferentiated anaplastic components were also observed in most carcinomas although at low percentages (Fig. 2B, S3, Tables S1, S2). The varied morphologic spectrum of tumor phenotypes and heterogeneity within individual tumors is a common feature of these models and
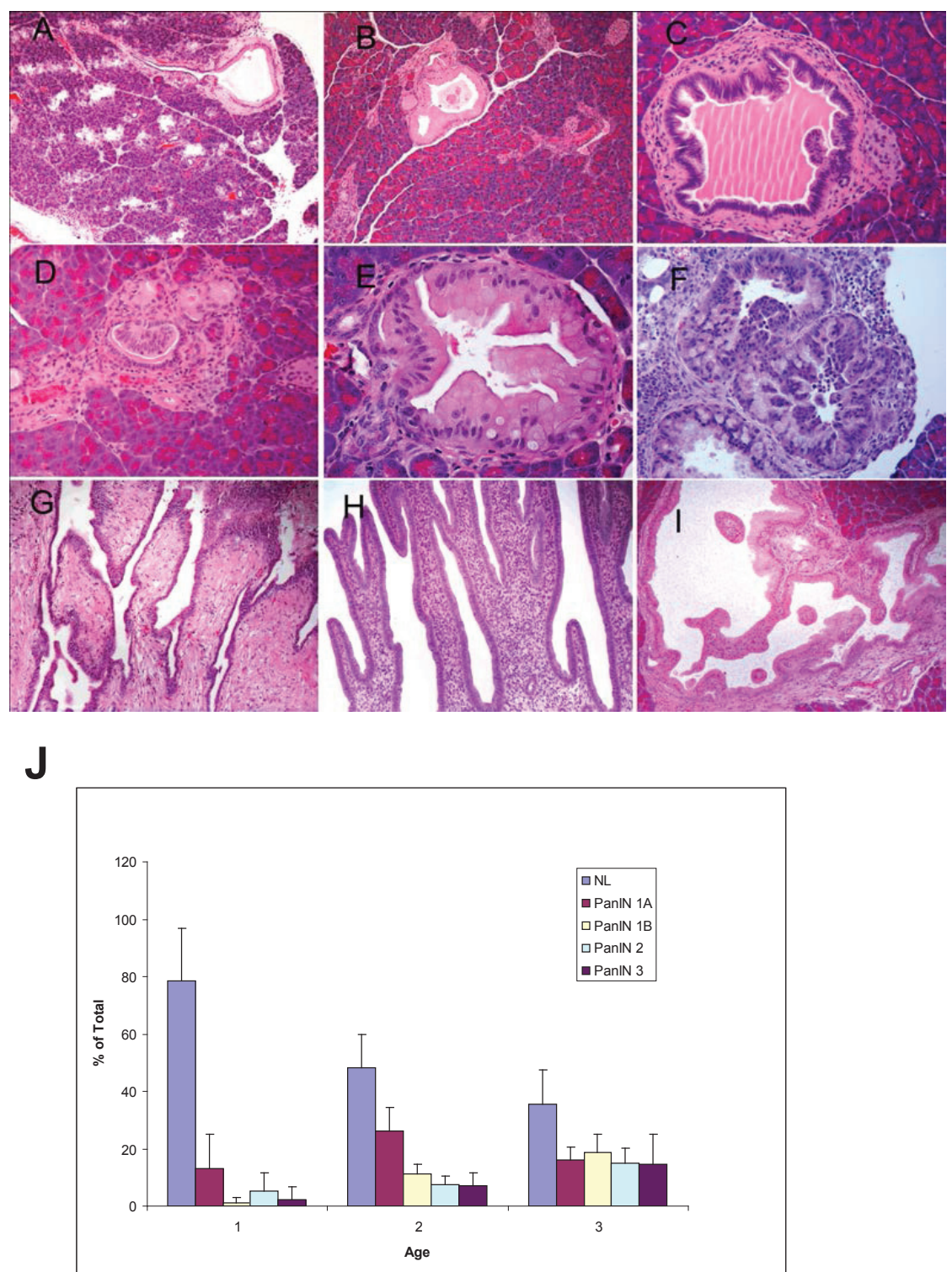

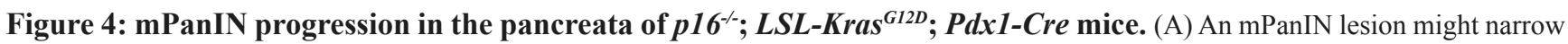
or obstruct the interlobular pancreatic duct (arrow); (B) mPanIN-1A with mild atypia (gastric foveolar type epithelium); (C) low-grade $\mathrm{mPanIN}-1 \mathrm{~B}$ to $\mathrm{mPanIN}-2$ lesion with mild cytologic atypia with focal micropapillary architecture; (D) focal mPanIN-2 lesion associated with background PanIN-1 lesion and (E) mPanIN-2 lesion with moderate cytologic atypia with focal micropapillary architecture; (F) High-grade mPanIN-3 lesion displays complete loss of cellular polarity, significant nuclear atypia, and budding of cell clusters into the ductal lumen. In addition to invasive tumors, pre-invasive occasional intraductal lesions with focal papillary $(\mathrm{G}, \mathrm{H})$ and cystic dilation (I), and multifocal mPanIN lesions with varying degrees of dysplasia were identified. (J) Histologic assessment of mPanIN progression in p16 ${ }^{--}$; LSL-Kras ${ }^{G 12 D}$; Pdxl-Cre mice. To quantify mPanIN progression, the total number of ducts, including normal and various stages of mPanINs, was scored in 3 age groups of $p 16^{-1} ; L S L-K r a s^{G 12 D} ; P d x 1$-Cre mice. Percentages ( \pm SEM) of normal (NL) and neoplastic ducts by grade $(\mathrm{mPanIN}-1 \mathrm{~A}, \mathrm{mPanIN}-1 \mathrm{~B}, \mathrm{mPanIN}-2$, and $\mathrm{mPanIN}-3)$ in the three groups are presented here. The average age for each group is cohort 1, 1.1 months $(n=8)$; cohort 2, 2.9 months $(n=5)$; and cohort 3, 4 months $(n=5)$. 
might result from the multifocal nature of the carcinomas. Invasive pancreatic adenocarcinomas expressed high levels of CK19 in well-differentiated regions and lower levels in sarcomatoid regions (Fig. 3A), whereas anaplastic regions showed no reactivity with anti-CK19. These anaplastic undifferentiated tumors had high mitotic activity, marked nuclear atypia, and dramatic cellular pleomorphism. The invasive carcinomas were usually associated with a desmoplastic stromal response, as shown by positive $\alpha$-SMA labeling of myofibroblasts (Fig. 3B). Ki67 immunoreactivity, which indicates cell proliferation, was significantly increased in PDA compared with normal pancreatic tissue (Fig. 3C). Increased p53 and p19 expressions were most frequently detected at the tumor margin, transit areas, and in well-differentiated PDA (Fig. 3D, E). Cyclin D1 and Fascin were also highly expressed by PDA (Fig. 3F, I). Immunoreactivity for Her2 and Cox-2 varied; they were restricted to a subset of PDA and undifferentiated sarcomatoid carcinoma (Fig. $3 \mathrm{G}, \mathrm{H})$. Immmunohistochemistry analyses were also performed on precancerous lesions. Increasing levels of immunoreactivity to Fascin, Ki67, Her-2, Cyclin D1, Cox2 were associated with $\mathrm{mPanIN}$ progression to invasive cancer as they are in humans (Table 1) [23], which further validated the similarities between the invasive pancreatic tumors found in our models and human PDA.

The invasive carcinomas that developed in $p 16$ - LSL-Kras ${ }^{G 12 D}$; Pdx1-Cre mice involved extensive amounts of peripancreatic tissue and frequently formed metastases. Numbers of metastases increased with age but were not restricted to older mice. Table $\mathrm{S} 1$ is a summary of $\mathrm{pl6}^{--}$; LSL- Kras ${ }^{G 12 D}$; Pdxl-Cre mutant mice that had been sacrificed by age for histology analyses. Metastases were identified in $44 \%$ of the mutant mice $(7 / 16$, Table S1) that were $<4$ months-old, $69 \%$ of those $>4$ months $(9 / 13)$. When mutant mice were sacrificed because they had reached the non-thriving stage, $100 \%$ had metastases $(n=14)$. Almost all of the non-thriving animals had overly distended abdomens, resulting from the accumulation of hemorrhagic ascites (Fig. S2). The distribution pattern of the metastases is similar to that of human disease. Most metastases involved the liver and regional lymph nodes (Fig. S4, Table S1), with direct local invasion of the duodenum, stomach, diaphragm, and spleen. Metastases to lymph nodes, liver, lung, and testis were also observed (Fig. S4). The mice frequently had obstructions of the bile duct or small bowel (Fig. S2). The liver metastases most frequently had a glandular morphology, with less frequent undifferentiated sarcomatoid and anaplastic components (Fig. S4). Therefore, not only the invasive pancreatic tumors observed in our models mimic human PDA, here we also report that the combined $p 16$ biallelic inactivation and Kras activation can promote frequent and consistent metastases that mimic the distribution pattern of metastases observed in advanced human PDA.

\section{Inactivation of p16 did not alter the histologic presentation of $\mathrm{mPanIN}$ initiated by oncogenic Kras}

Tumors from $p 16^{-\sim}$; LSL- Kras ${ }^{G 12 D} ;$ Pdxl-Cre mice were collected from mice at 4-6, 11-13 and 15-17 weeks and histologic analyses were performed. A full spectrum of mPanIN lesions were observed in the p16 $6^{--} L S L$ Kras $^{G 12 D}$; Pdx1-Cre mice (Fig. $4 \mathrm{~B}-\mathrm{F}$ ), indicating that the biallelic inactivation of $p 16$ accelerated but did not alter the pathological progression of the $L S L-\mathrm{Kras}^{G 12 D}$; Pdxl-Cre mice [11]. At $\sim 1$ month of age, 5/8 p16 LSL-Kras ${ }^{G 12 D}$; Pdxl-Cre mice had focal mPanIN lesions (predominantly low grade) but no PDA. The remaining 3 had normal pancreatic histology (Fig. 4J). At 3-4 months, p16 $6^{-1}$;SL- Kras ${ }^{G 12 D} ; \mathrm{Pdxl}$-Cre mice had a significantly increased overall number and advanced grade of mPanINs compared with 1-month old mice (Fig. 4J). Mutant mice that were $>3$ months old all had multifocal PDA. Highgrade $\mathrm{mPanIN}-3$ lesions were frequently associated with PDA. The control littermates had normal pancreatic histology at each time point. Infrequently, focal and noninvasive cystic papillary neoplasms, resembling human intraductal papillary mucinous neoplasms were also observed (Fig. 4G-I) [24].

Similar histological progression pattern from precancerous lesions to invasive cancer and metastasis was also observed in the $p 16^{f f x} f f o x ; L S L-\mathrm{Kras}^{G 12 D} ; \mathrm{Pdxl}$-Cre mice (Fig. S3), again demonstrating that p16 inactivation promotes progression but does not alter tumor initiation

\section{Table 1: Immunohistochemical profile of the precancerous lesions and pancreatic ductal

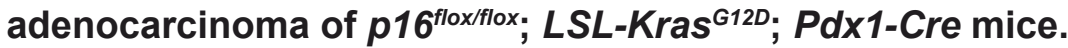

\begin{tabular}{|l|c|c|c|c|c|}
\hline Marker & Normal & PanlN-1* & PanlN-2 & PanlN-3 & PDA* $^{*}$ \\
\hline Fascin & - & $+/-$ & ++ & ++ & +++ \\
\hline Ki67 & $+/-$ & $+/-$ & + & ++ & ++ \\
\hline Her-2 & - & - & + & ++ & ++ \\
\hline Cyclin D1 & - & $+/-$ & + & ++ & +++ \\
\hline Cox-2 & - & - & + & ++ & ++ \\
\hline * PanIN, pancreatic intrae pithelial neoplasia; PDA, pancreatic ductal adenocarcinoma &
\end{tabular}


A

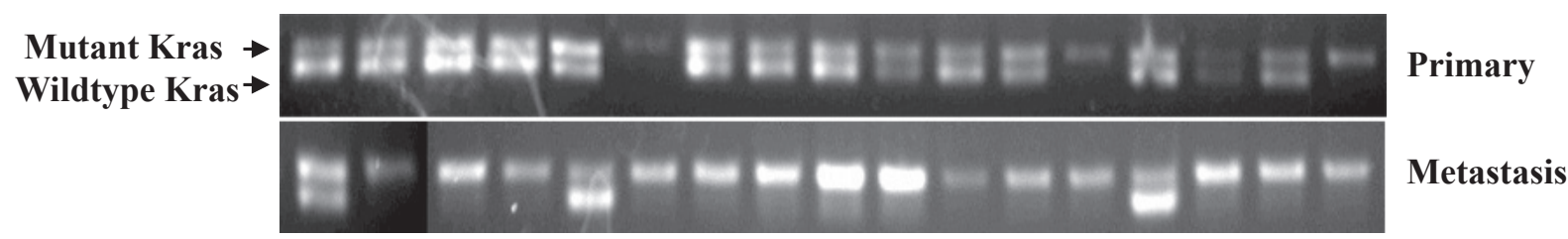

B

C


D

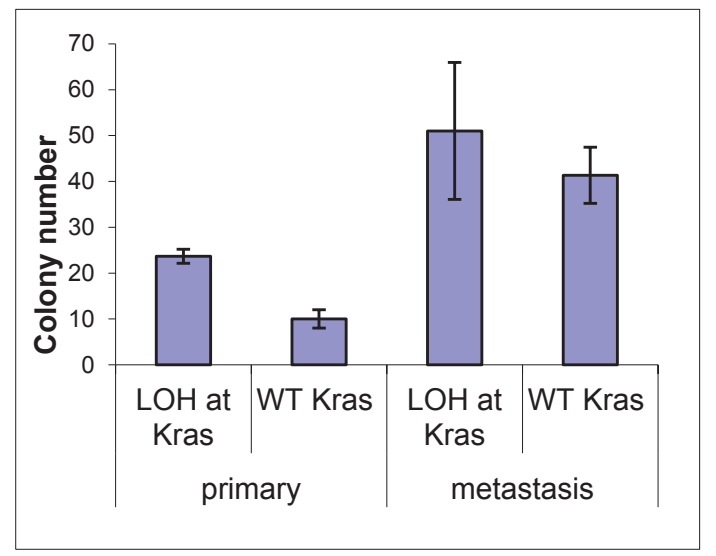

E
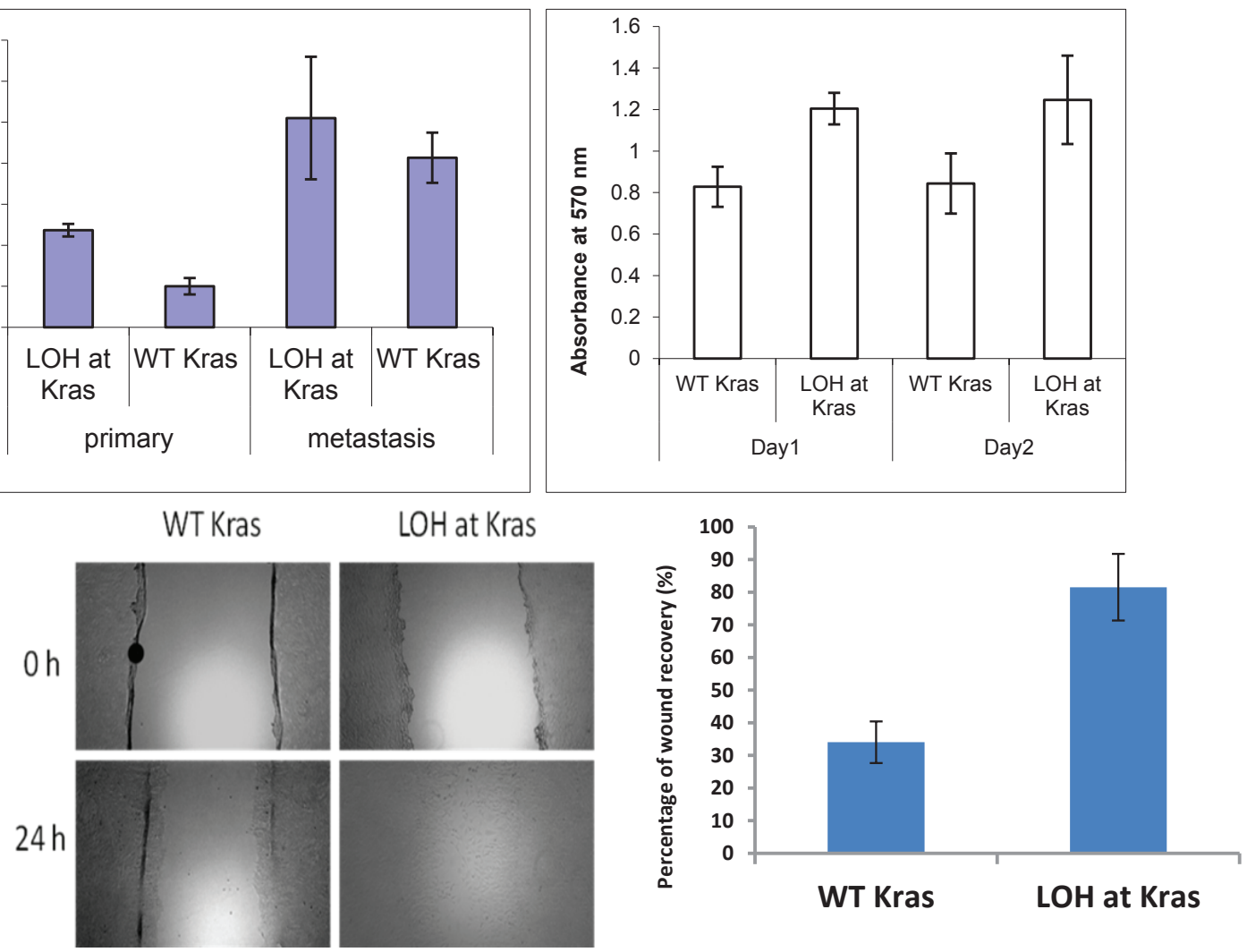

Figure 5: Loss of the wild-type allele of Kras is associated with invasiveness and metastasis of pancreatic tumors in p16 $^{f o x / f l o x}$; LSL-Kras ${ }^{G 12 D} ;$ Pdx1-Cre mice. (A) Representative samples showing loss of the wild-type allele of Kras (LOH at Kras) occurred more frequently in cancer cell lines derived from metastases than those derived from primary tumors of $p 16^{\text {fox } x \text { fox }}$; LSL-Kras ${ }^{\text {GI2D; }}$; Pdxl-Cre mice by PCR of genomic DNA, and confirmed by. (B) LOH at Kras was confirmed by RT-PCR and restriction fragment length polymorphism analyses [18]. PCR-amplified cDNA was untreated (-) or digested with HindIII (+). Representative pancreatic cancer cell lines 1 to 3 contained both wild-type (upper band) and mutant Kras alleles (lower band), whereas cell lines 4 and 5 lacked the wild-type Kras allele. (C, D) In colony formation assay with soft agar, primary pancreatic cancer cell lines with LOH at Kras formed significantly more colonies $(23.7 \pm 1.5)$ than those retained the Kras allele $(10.0 \pm 2.0)(\mathrm{p}<0.05)$. There was no significant difference between metastatic cells with or without $\mathrm{LOH}$ at $\operatorname{Kras}(51.0 \pm 14.9$ vs. $41.3 \pm 6.1 ; \mathrm{P}>0.05)$. (E) LOH at Kras also promoted cellular proliferation of the primary cancer cell lines in low serum environment at day $1(\mathrm{P}<0.01)$ and day $2(\mathrm{p}<0.05)$ by MTT assay. $(\mathrm{F}, \mathrm{G})$ Increased migration was also correlated with $\mathrm{LOH}$ at Kras as measured by percentage of wound healing at 24 hours. The data represent the mean $\pm \mathrm{SD}(\mathrm{n}=3, \mathrm{p}<0.05)$. 
and histology.

\section{Loss of the wild-type Kras allele promotes pancreatic tumor cell proliferation and metastasis in mice and humans}

Primary cell cultures were established from 12 primary tumors and 2 metastases of the $p 16^{\text {fox } f f o x}$; $L S L-K_{r a s}{ }^{G 12 D}$; Pdxl-Cre mice (Two of the 12 primary tumors and the two metastases were harvested from the same mice). From these primary cell cultures, we further established 464 clonal cancer cell lines from the 12 pancreatic primary tumors and 42 clonal cancer cell lines from the metastasis. Smad4 and p19 proteins were detected in all the clonal cancer cell lines examined (Fig. S5). We sequenced $p 53$ cDNA of $\sim 100$ clonal cancer cell lines but did not uncover any $p 53$ mutation in these cells.

$L S L-\operatorname{KraS}^{G 12 D}$ was conditionally activated in all of the pancreatic cancer cell lines (Fig. 5A), indicating that Kras activation is required for pancreatic tumorigenesis in these mice. Surprisingly, some of the cell lines possessed only the recombined Kras allele, but not the wild-type Kras allele (Fig. 5A). RT-PCR/RFLP analysis of RNA confirmed that the wild-type allele of Kras was lost ( $\mathrm{LOH}$ at Kras) in some tumor cell lines but never the mutant $\mathrm{Kras}^{G 12 \mathrm{D}}$ allele (Fig. 5B). Comparing the cell lines derived from the two paired primary tumors and metastases derived from the same mice, the frequency of $\mathrm{LOH}$ at $\mathrm{Kras}$ was greater among the cancer cell lines derived from each metastasis $(8.3 \%$ and $33.3 \%)$ than those derived from the corresponding primary pancreatic tumor $(0 \%$ and $17.6 \%$, respectively) ( $\mathrm{p}=0.16$ and $\mathrm{p}<0.05$, respectively) (Fig. $5 \mathrm{~A}$ ), suggesting Kras LOH might promote metastasis in vivo. We also microdissected liver metastases to confirm that LOH at Kras indeed occurred in vivo, and was not an artifact generated by tissue culture adaptation (Fig. S6).

To investigate if $\mathrm{LOH}$ at Kras has any phenotypic consequences, cancer cell lines derived from the primary tumor and metastasis of the same $p 16^{f 0 x f f o x} ; L S L-K_{r a s}^{G 12 D}$; $\mathrm{Pdxl-Cre}$ mouse were subjected to in vitro assays. In soft-agar colony formation assays, clonal cell lines of the primary tumor with $\mathrm{LOH}$ at Kras $(23.7 \pm 1.5)$ formed more colonies than those without LOH at Kras (10.0 2.0$)$ $(p<0.05)$. The numbers of colonies formed by cell lines from the metastasis were generally greater than those of the primary tumor cells, but the difference between the metastatic cell lines with or without $\mathrm{LOH}$ at $\mathrm{Kras}$ (51.0 014.9 vs. $41.3 \pm 6.1$ respectively) was not statistically significant (Fig. 5C, D). Under low serum condition, significant increased proliferation was observed in primary tumor cell lines with $\mathrm{LOH}$ at Kras than those without (Fig. 5E). Loss of the wild-type Kras allele also promoted motility among the primary cancer cell lines significantly (Fig 5F, G). Real-time PCR did not detect amplification of the mutant Kras allele in primary or metastatic cancer cell lines and confirmed the loss of the wild-type Kras allele (Table S3); indicating that the functional differences observed in these cancer cell lines were not caused by

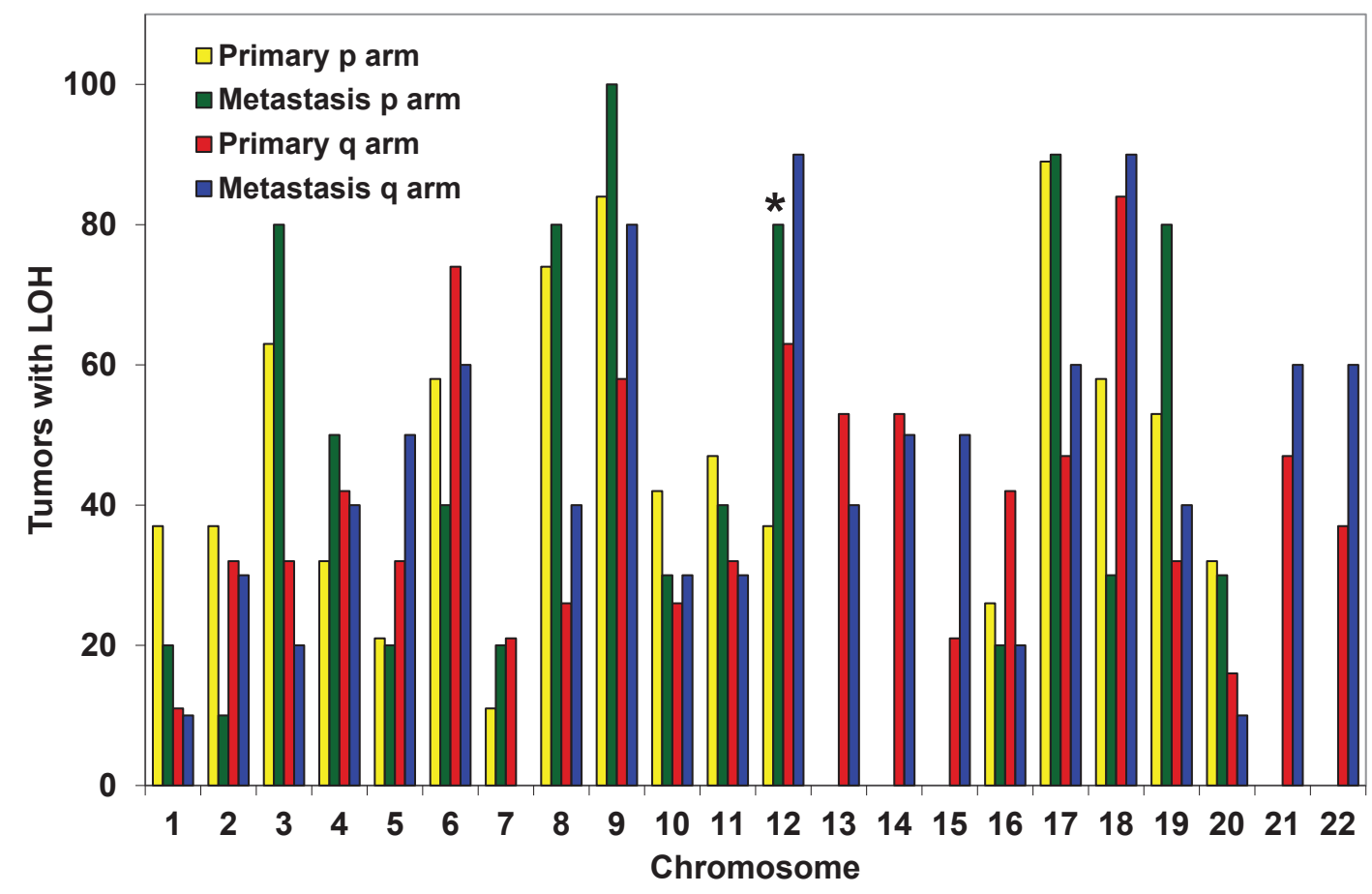

Figure 6: Increased LOH at KRAS (chromosome 12p) in human metastases compared with primary pancreatic cancers. SNP analyses were performed on human cancer cell lines derived from pancreatic primary tumors $(n=19)$ or metastases $(n=10)$. High frequency allelic loss was exclusively observed at chromosome $12 \mathrm{p}$ (where KRAS is located) in metastatic cells, but not primary tumor cells $(\mathrm{p}<0.02)$. 
differential mutant Kras amplification, but rather by the loss of the wild-type Kras allele. We investigated whether the MAPK signaling pathway is responsible for the functional differences observed in primary and metastatic cancer cell lines with or without LOH at Kras and found no discernible difference of ERK1/2 phosphorylation in cancer cell lines with or without LOH of Kras by western blot analyses.

Our in vivo and in vitro data suggested that $\mathrm{LOH}$ at Kras rendered primary pancreatic tumor cells more aggressive functional phenotypes that favored growth and metastasis in mice. To investigate if such phenomena also occurs in humans, non-biased whole-genome LOH profiles were generated from human cancer cell lines derived from primary pancreatic tumors $(n=19)$ or metastases $(n=10)$. There was no statistical difference in the $\mathrm{LOH}$ profiles of the primary tumors $v s$. metastases except at chromosome $12 \mathrm{p}$, which contains the $K R A S$ gene. $\mathrm{LOH}$ at chromosome $12 \mathrm{p}$ was observed in $37 \%$ of primary and $80 \%$ of metastatic cancer cell lines $(\mathrm{p}<0.02)$ (Fig. 6). LOH at KRAS was confirmed by genomic sequencing of the KRAS gene in the cancer cell lines with LOH at chromosome 12p (Fig. S7). The lack of significant difference in the allelic loss on other chromosomes strongly supports that the increased $\mathrm{LOH}$ at chromosome $12 \mathrm{p}$ is a selective and targeted event. These data corroborates the observations made in our mice, suggesting that $\mathrm{LOH}$ at $K R A S$ is associated with metastasis and may promote metastasis.

\section{DISCUSSION}

We reported the first mouse line with conditional knockout of $p 16$ that does not alter $p 19$ expression (Fig. 1 , S6). This mouse strain is distinct from the previously reported $p 16 / p 19$ knockout strains $[18,21]$. These mice are useful for investigating the functions of $\mathrm{p} 16$, without disrupting the p19-Mdm2-p53 pathway, in tissue-specific manners.

In combination with expression of $\operatorname{Kras}^{G 12 D}$, pancreasspecific disruption of 16 in $L S L-\mathrm{Kras}^{G 12 D}$; Pdx1-Cre mice promoted progression of $\mathrm{mPanIN}$ to invasive pancreatic ductal adenocarcinoma and significantly shorten their median survival from $>13$ months to 4-6 months (Fig. 2) [25]. The $p 16^{--} ;$LSL-Kras ${ }^{G 12 D} ;$ Pdx1-Cre mice and $p 16^{\text {flox }}$ flox; LSL-Kras ${ }^{G 12 D}$; Pdx1-Cre mice had median survival times of 4 and 6 months respectively-longer than the 2-month average survival time reported for $p 16 / p 19^{\text {flox } f l o x}$; LSL-Kras ${ }^{G 12 D}$; Pdx1-Cre and p16/p19-- ; LSL- Kras ${ }^{G 12 D}$; Pdx1-Cre mice $[18,21]$. The survival curves of $p 16 /$ p19foxfflox; LSL-Kras ${ }^{G 12 D} ; \mathrm{Pdx} 1-\mathrm{Cre}$ and p16/p19-/ ; LSLKras $^{G 12 D}$; Pdx1-Cre mice more closely resemble those of p53 floxfflox; LSL-Kras ${ }^{G 12 D} ;$ Pdx1-Cre mice, perhaps due to the fact that $p 19$ and $p 53$ belong in the same pathway. Therefore it is important to differentiate the contributions of $p 16$ and $p 19$ to pancreatic tumorigenesis.

Similar mPanIN progression profile was observed in $p 16^{--} ; L S L-K_{r a s}^{G 12 D} ; P d x 1-C r e$ and $p 16^{\text {floxfflox }}$; LSLKras $^{G 12 D}$; Pdx1-Cre mice as in LSL-Kras ${ }^{G 12 D}$; PDX1-Cre mice but at an accelerated pace (Fig. 4), indicating that biallelic inactivation of $p 16$ accelerated the development of PDA in synergy with $\operatorname{Kras}^{G 12 D}$ but did not alter the pathological progression of the pancreatic lesions- $20 \%$ of the pancreatic ducts in $p 16^{--} ; L S L-K_{r a s}^{G 12 D} ; P d x 1-$ Cre mice had mPanIN presentations around 4 weeks of age (Fig. 4), as opposed to $>2$ months in LSL-Kras ${ }^{G 12 D}$; PDX1-Cre mice [11]. The mPanIN lesions evolved and progressed to invasive cancer, and the most common histologic variant was comprised predominantly of malignant glands characterizing a well to moderately differentiated adenocarcinoma (Fig. 2, S3, Tables S1, S2). This is in contrast to the undifferentiated sarcomatoid histology previously reported in $p 16^{--} ; L S L-K_{r a s}^{G 12 D}$; Pdx1-Cre mice $(\mathrm{n}=3)$ [21]. The difference may simply be in the number of mice examined, but nevertheless, given that the majority of human pancreatic cancer is presented as well to moderately differentiated ductal adenocarcinoma, it's important that we have demonstrated here that biallelic inactivation of p16 in conjunction with oncogenic Kras in mice mimic human pancreatic tumor progression from PanIN to PDA.

A striking feature of the $p 16^{--} ; L S L-K_{r a s}^{G 12 D} ; P d x 1$ Cre and $p 16^{\text {floxflox }}$; LSL-Kras ${ }^{G 12 D}$; Pdx1-Cre mice was the high penetrance of metastases. Metastases were observed in $100 \%$ of the non-thriving $p 16^{--} ; L S L-K_{r a s}^{G 12 D} ; P d x 1-$ Cre mice $(\mathrm{n}=14)$. It has been reported previously that only $63 \%$ of $p 53^{R 172 H /+}$; LSL-Kras $S^{G 12 D}$; PDX1-Cre mice developed metastasis [25, 26], 33\% was detected in p53 $3^{\text {lox/+}}$; LSL-Kras ${ }^{G 12 D}$; PDX1-Cre mice, and none was reported in p53 lox/lox :LSL-Kras ${ }^{G 12 D}$; PDX1-Cre mice [21, 26]. It appears that inactivation of $p 16$ in the presence of activated Kras is associated with increased metastasis in comparison to $p 53$ inactivation; however, the mechanism behind this difference is not known presently. This difference in metastasis frequency cannot be explained by mere difference in medium survival- the reported medium survival of $p 53^{R 172 H /+} ; L S L-K r a s S^{G 12 D}$; PDX1-Cre mice (5 months) [25] is longer than that of $p 16^{-/}$; LSL- Kras ${ }^{G 12 D}$; $P d x 1$-Cre mice. The frequent and consistent metastases observed in the $p 16^{-/} ; L S L-K r a s^{G 12 D} ; P d x 1-C r e$ and $p 16^{\text {flox }}$ ${ }^{f l o x}$; LSL-Kras ${ }^{G 12 D}$; Pdx1-Cre mice make them ideal models for studying advanced human PDA and metastasis.

Numerous studies in mouse models have shown that $\operatorname{Kras}^{G 12 D}$ is sufficient to initiate development of PanINs and their progression to invasive cancer $[6,10,11]$. Here we show that that in addition to its role in tumor initiation, Kras ${ }^{G 12 D}$ may promote tumor progression and metastasis. The loss of the wild-type allele of Kras during the progression from primary carcinomas to metastases suggests that its loss might confer growth advantage in the presence of oncogenic e $\mathrm{Kras}^{G 12 D}$ allele. LOH at Kras did not appear to be a random event, because only the wildtype Kras allele was selectively lost, which resulted in 
discernible functional phenotypes (Fig. 5). LOH at Kras was detectable in liver metastases and not a mere result of tissue culture adaptation (Fig. S6). LOH at Kras was not an artifact created by genetic engineering or unique to mice, because an increased frequency of LOH at KRAS was observed in human metastatic cell lines (80\%) compared with primary cancer cell lines (37\%) (Fig. 6). KRAS is located on chromosome $12 \mathrm{p}$ - the only chromosomal region in which a statistically significant difference was observed between human primary and metastatic cancer cells. The absence of increased $\mathrm{LOH}$ at other chromosomal arm strongly supports that the $\mathrm{LOH}$ at $K R A \mathrm{~S}$ is not simply a manifestation of increased genomic stability during progression. Previous studies proposed that a wild-type allele of Kras allele can serve as a tumor-suppressor [7, 12, 27-30]. Our data demonstrate that sporadic loss of the wild-type Kras allele occurred in pancreatic tumors and metastases in both mice and humans. Our data is the first to provide in vivo and in vitro evidence that $\mathrm{LOH}$ at wildtype Kras is associated with pancreatic tumor metastasis.

The functional impacts of oncogenic Kras in tumor initiation are well known, but the potential role of wildtype Kras in this process remains elusive. It is possible that the loss of wild-type Kras merely serves to remove a competitor for limited resources, such as GTP. However, many previous studies have suggested that the dynamics between the wild-type and mutant Kras proteins is complex and the expression level of the mutant Kras allele and/ or the ratio of the wild-type to mutant Kras proteins are critical to tumorigenesis $[6-10,12,13]$. Identification of differential downstream effector proteins for wild-type and oncogenic KRAS in the future will help elucidating if the wild-type $K R A S$ allele possesses any tumor-suppressive function. However, regardless what the underlying molecular mechanism is, our current study already has a strong implication on the development KRAS target therapy. The current thinking on KRAS target therapy does not differentiate between inhibiting wild-type or oncogenic KRAS. Our data strongly recommends that future KRAS target therapies should specifically inhibit only the oncogenic KRAS, not the wild-type KRAS, because the non-discriminatory inhibition of wild-type KRAS may promote metastasis and produce unintended negative impacts on patient care.

We show that biallelic inactivation of the p16 and $\mathrm{Kras}^{G 12 D}$ act synergistically to promote pancreatic tumorigenesis and metastasis. The p16 foxffox; LSLKras ${ }^{G 12 D}$; Pdx1-Cre mice develop PanIN, invasive tumors, and metastases in a manner that recapitulates human PDA development and progression. The relative short median survival and high incidence of metastasis in these mice make them an attractive model for testing novel therapeutics and investigating metastasis -2 areas of research that are a challenge to explore in humans.

\section{MATERIALS AND METHODS}

\section{Genetically engineered mouse models and mouse strains}

To generate a conditional p16 knockout mouse line $\left(p 16^{f 0 x f f l o x}\right)$, exon $1 \alpha$ of $p 16$ was flanked by loxP sites, leaving $p 19$ expression intact (Fig. S1 and 1). The details on the generation of the $p 16^{\text {fox } f \text { fox }}$ mouse line are described in the Supplementary Methods. The resultant $p 16^{f f x x f f o x}$ mice were of mixed $129 / \mathrm{SvJ}$ and C57BL/6 backgrounds. The $p 16^{-1-}[22]$ and $L S L-K_{r a s}^{G 12 D}$ [11] mice were obtained through the Mouse Models of Human Cancers Consortium Repository. Pdxl-Cre mice were previously described [11]. All studies were conducted in compliance with the IACUC guidelines of Columbia University.

\section{Histology and immunohistochemistry}

Tissues were fixed in 10\% formalin overnight and embedded in paraffin. The detailed protocol and antibodies for immunohistochemistry are described in the Supplementary Methods.

\section{Establishment and cultivation of primary pancreatic adenocarcinoma cell lines}

Freshly isolated murine tumor specimens were minced with sterile razor blades, digested with $0.5 \%$ trypsin for 15 minutes at $37^{\circ} \mathrm{C}$, resuspended in DMEM and $15 \%$ fetal bovine serum (FBS), and seeded in 10 $\mathrm{cm}$ dishes. All genetic analyses were performed on cells cultivated for less than 7 passages. Each primary cancer cell culture was then sorted by flow cytometry and seeded at a single cell per well, into 96-well tissue-culture plates, to establish clonal cell lines. About 18 to 50 clonal cell lines were obtained from each original primary cancer cell line.

\section{Immunoblot analysis}

Tissues or cell pellets were lysed in $20 \mathrm{mM}$ Tris $(\mathrm{pH}$ 7.5), $150 \mathrm{mM} \mathrm{NaCl}, 1 \mathrm{mM}$ EDTA, 1 $\mathrm{mM}$ EGTA, and 1\% Triton X-100 in the presence of a protease inhibitor cocktail (Roche, Indianapolis, IN) or and a phosphatase inhibitor cocktail (kits I and II, Calbiochem). Immunoblotting was performed as described previously [31,32] and as in the Supplementary Methods.

\section{Colony formation, MTT, and migration assays}

These assays were performed with standard protocols and briefly described in the Supplementary Methods. All experiments were performed in triplicate and repeated at 
least 3 times.

\section{SNP analyses of human cancer cell lines}

Twenty-nine human cancer cell lines were used, and segregated into those arising from primary carcinomas (BxPc3, MiaPaCa-2, Panc-1, PK9, PL-1, PL-5, PL-9, PL-11, PL-13, PL-19, PL20, PL21, PL22, PL23, PL24, XPA1, XPA2, XPA3, and A13A/B) or from pancreatic cancer metastases (AsPc1, CAPAN1, CFPAC1, Hs766T, PK-8, Su86.86, A6L, A2.1, A13D, and A10.7) [33, 34]. The allelotypes of 24 of these cell lines were described previously [33], whereas those of cell lines A13A/B, A6L, A2.1, A13D and A10.7 were generated for this study. The genotypes of 115,353 SNPs were analyzed in all cell lines using Affymetrix oligonucleotide arrays hybridized to reduced complexity genomic DNA as previously described [35]. Genotypes were determined by the GeneChip® DNA Analysis Software Tool (GDAST, v3.0) using a 0.05 quality score setting genotypes were plotted with respect to their genomic position listed in the May 2004 assembly of the human genome and briefly described in the Supplementary Method.

\section{Statistical analysis}

Results were presented as the mean \pm SD. the Student $t$ test was used to compare data between groups and the $\chi 2$ method was used to compare the frequency of allelic loss among cell lines. $\mathrm{P}$ values less than 0.05 were considered to be statistically significant.

\section{ACKNOWLEDGEMENTS}

We sincerely thank Dr. Richard L. Huganir and Dr. Kogo Takamiya and at The Johns Hopkins University for their generosity in sharing their designed vectors and cloning strategies. This work was supported by the Michael Rolfe Foundation, the Lustgarten Foundation, NCI Temin Award CA95434 and NCI R01 CA109525.

\section{REFERENCES}

1. Jemal A, Siegel R, Ward E, Hao Y, Xu J, Thun MJ: Cancer statistics, 2009. CA Cancer J Clin 2009, 59(4):225-249.

2. Kern S, Hruban R, Hollingsworth MA, Brand R, Adrian TE, Jaffee E, Tempero MA: A white paper: the product of a pancreas cancer think tank. Cancer Res 2001, 61(12):49234932.

3. Almoguera C, Shibata D, Forrester K, Martin J, Arnheim N, Perucho M: Most human carcinomas of the exocrine pancreas contain mutant c-K-ras genes. Cell 1988, 53(4):549-554.

4. Smit VT, Boot AJ, Smits AM, Fleuren GJ, Cornelisse CJ,
Bos JL: KRAS codon 12 mutations occur very frequently in pancreatic adenocarcinomas. Nucleic Acids Res 1988, 16(16):7773-7782.

5. Hruban RH, Wilentz RE, Kern SE: Genetic progression in the pancreatic ducts. Am J Pathol 2000, 156(6):1821-1825.

6. Guerra C, Mijimolle N, Dhawahir A, Dubus P, Barradas M, Serrano M, Campuzano V, Barbacid M: Tumor induction by an endogenous K-ras oncogene is highly dependent on cellular context. Cancer Cell 2003, 4(2):111-120.

7. Zhang Z, Wang Y, Vikis HG, Johnson L, Liu G, Li J, Anderson MW, Sills RC, Hong HL, Devereux TR et al: Wildtype Kras2 can inhibit lung carcinogenesis in mice. Nat Genet 2001, 29(1):25-33.

8. Brembeck FH, Schreiber FS, Deramaudt TB, Craig L, Rhoades B, Swain G, Grippo P, Stoffers DA, Silberg DG, Rustgi AK: The mutant K-ras oncogene causes pancreatic periductal lymphocytic infiltration and gastric mucous neck cell hyperplasia in transgenic mice. Cancer Res 2003, 63(9):2005-2009.

9. Grippo PJ, Nowlin PS, Demeure MJ, Longnecker DS, Sandgren EP: Preinvasive pancreatic neoplasia of ductal phenotype induced by acinar cell targeting of mutant kras in transgenic mice. Cancer Res 2003, 63(9):2016-2019.

10. Tuveson DA, Shaw AT, Willis NA, Silver DP, Jackson EL, Chang S, Mercer KL, Grochow R, Hock H, Crowley $D$ et al: Endogenous oncogenic K-ras(G12D) stimulates proliferation and widespread neoplastic and developmental defects. Cancer Cell 2004, 5(4):375-387.

11. Hingorani SR, Petricoin EF, Maitra A, Rajapakse V, King C, Jacobetz MA, Ross S, Conrads TP, Veenstra TD, Hitt $\mathrm{BA}$ et al: Preinvasive and invasive ductal pancreatic cancer and its early detection in the mouse. Cancer Cell 2003, 4(6):437-450.

12. Li J, Zhang Z, Dai Z, Plass C, Morrison C, Wang Y, Wiest JS, Anderson MW, You M: LOH of chromosome $12 \mathrm{p}$ correlates with Kras 2 mutation in non-small cell lung cancer. Oncogene 2003, 22(8):1243-1246.

13. To MD, Wong CE, Karnezis AN, Del Rosario R, Di Lauro $\mathrm{R}$, Balmain A: Kras regulatory elements and exon 4A determine mutation specificity in lung cancer. Nat Genet 2008, 40(10):1240-1244.

14. Jones S, Zhang X, Parsons DW, Lin JC, Leary RJ, Angenendt P, Mankoo P, Carter H, Kamiyama H, Jimeno A et al: Core signaling pathways in human pancreatic cancers revealed by global genomic analyses. Science 2008, 321(5897):1801-1806.

15. Schutte M, Hruban RH, Geradts J, Maynard R, Hilgers W, Rabindran SK, Moskaluk CA, Hahn SA, SchwarteWaldhoff I, Schmiegel W et al: Abrogation of the Rb/ p16 tumor-suppressive pathway in virtually all pancreatic carcinomas. Cancer Res 1997, 57:3126-3130.

16. Rozenblum E, Schutte M, Goggins M, Hahn SA, Lu J, Panzer S, Zahurak M, Goodman SN, Hruban RH, Yeo CJ et al: Tumor-suppressive pathways in pancreatic carcinoma. 
Cancer Res 1997, 57:1731-1734.

17. Serrano M, Lee H, Chin L, Cordon-Cardo C, Beach D, DePinho RA: Role of the INK4a locus in tumor suppression and cell mortality. Cell 1996, 85(1):27-37.

18. Aguirre AJ, Bardeesy N, Sinha M, Lopez L, Tuveson DA, Horner J, Redston MS, DePinho RA: Activated Kras and Ink4a/Arf deficiency cooperate to produce metastatic pancreatic ductal adenocarcinoma. Genes Dev 2003, 17(24):3112-3126.

19. Lowe SW, Sherr CJ: Tumor suppression by Ink4a-Arf: progress and puzzles. Curr Opin Genet Dev 2003, 13(1):7783.

20. Pomerantz J, Schreiber-Agus N, Liegeois NJ, Silverman A, Alland L, Chin L, Potes J, Chen K, Orlow I, Lee HW et al: The Ink4a tumor suppressor gene product, p19Arf, interacts with MDM2 and neutralizes MDM2's inhibition of p53. Cell 1998, 92(6):713-723.

21. Bardeesy N, Aguirre AJ, Chu GC, Cheng KH, Lopez LV, Hezel AF, Feng B, Brennan C, Weissleder R, Mahmood $\mathrm{U}$ et al: Both p16(Ink4a) and the p19(Arf)-p53 pathway constrain progression of pancreatic adenocarcinoma in the mouse. Proc Natl Acad Sci U S A 2006, 103(15):59475952.

22. Sharpless NE, Bardeesy N, Lee KH, Carrasco D, Castrillon DH, Aguirre AJ, Wu EA, Horner JW, DePinho RA: Loss of p16Ink4a with retention of p19Arf predisposes mice to tumorigenesis. Nature 2001, 413(6851):86-91.

23. Maitra A, Adsay NV, Argani P, Iacobuzio-Donahue C, De Marzo A, Cameron JL, Yeo CJ, Hruban RH: Multicomponent analysis of the pancreatic adenocarcinoma progression model using a pancreatic intraepithelial neoplasia tissue microarray. Modern Pathology 2003, 16(9):902-912.

24. Maitra A, Fukushima N, Takaori K, Hruban RH: Precursors to invasive pancreatic cancer. Adv Anat Pathol 2005, 12(2):81-91.

25. Hingorani SR, Wang L, Multani AS, Combs C, Deramaudt TB, Hruban RH, Rustgi AK, Chang S, Tuveson DA: Trp53R172H and KrasG12D cooperate to promote chromosomal instability and widely metastatic pancreatic ductal adenocarcinoma in mice.[see comment]. Cancer Cell 2005, 7(5):469-483.

26. Morton JP, Timpson P, Karim SA, Ridgway RA, Athineos D, Doyle B, Jamieson NB, Oien KA, Lowy AM, Brunton VG et al: Mutant p53 drives metastasis and overcomes growth arrest/senescence in pancreatic cancer. Proc Natl Acad Sci U S A, 107(1):246-251.

27. Hegi ME, Devereux TR, Dietrich WF, Cochran CJ, Lander ES, Foley JF, Maronpot RR, Anderson MW, Wiseman RW: Allelotype analysis of mouse lung carcinomas reveals frequent allelic losses on chromosome 4 and an association between allelic imbalances on chromosome 6 and K-ras activation. Cancer Res 1994, 54(23):6257-6264.

28. Bremner R, Balmain A: Genetic changes in skin tumor progression: correlation between presence of a mutant ras gene and loss of heterozygosity on mouse chromosome 7 . Cell 1990, 61(3):407-417.

29. Li H, Cao HF, Wan J, Li Y, Zhu ML, Zhao P: Growth inhibitory effect of wild-type Kras2 gene on a colonic adenocarcinoma cell line. World J Gastroenterol 2007, 13(6):934-938.

30. Fleming JB, Shen GL, Holloway SE, Davis M, Brekken RA: Molecular consequences of silencing mutant K-ras in pancreatic cancer cells: justification for K-ras-directed therapy. Molecular Cancer Research: MCR 2005, 3(7):413423.

31. Qiu W, Schonleben F, Thaker HM, Goggins M, Su GH: A novel mutation of STK11/LKB1 gene leads to the loss of cell growth inhibition in head and neck squamous cell carcinoma. Oncogene 2006.

32. Qiu W, Schonleben F, Li X, Su GH: Disruption of transforming growth factor beta-Smad signaling pathway in head and neck squamous cell carcinoma as evidenced by mutations of SMAD2 and SMAD4. Cancer Lett 2007, 245(1-2):163-170.

33. Calhoun ES, Hucl T, Gallmeier E, West KM, Arking DE, Maitra A, Iacobuzio-Donahue CA, Chakravarti A, Hruban $\mathrm{RH}$, Kern SE: Identifying allelic loss and homozygous deletions in pancreatic cancer without matched normals using high-density single-nucleotide polymorphism arrays. Cancer Research 2006, 66(16):7920-7928.

34. Fu B, Guo M, Wang S, Campagna D, Luo M, Herman JG, Iacobuzio-Donahue CA: Evaluation of GATA-4 and GATA-5 methylation profiles in human pancreatic cancers indicate promoter methylation patterns distinct from other human tumor types. Cancer Biol Ther 2007, 6(10):15461552.

35. Matsuzaki H, Dong S, Loi H, Di X, Liu G, Hubbell E, Law J, Berntsen T, Chadha M, Hui H et al: Genotyping over 100,000 SNPs on a pair of oligonucleotide arrays. Nat Methods 2004, 1(2):109-111. 